\title{
SEGMENTATION OF STROKE REGIONS FROM DWI AND ADC SEQUENCES USING A MODIFIED WATERSHED METHOD
}

\author{
Ravi S. ${ }^{1}$, A.M. Khan ${ }^{2}$ \\ ${ }^{I}$ Research Student, Dept. of Electronics, Mangalore University, Mangalagangotri, India \\ ${ }^{2}$ Professor \& Chairman, Dept. of Electronics, Mangalore University, Mangalagangotri, India
}

\begin{abstract}
Stroke is a cerebro-vascular accident that alters the supply of blood to the brain cells. Stroke is second highest cause for morbidity rate among humans after cardiovascular arrest. Stroke can hardly be observed in T1 and T2 sequences of MRI on the onset. The doctors usually prefer diffusion weighted imaging sequence and apparent diffusion coefficient mapping to diagnose strokes. Segmentation is the necessary part of medical image processing to get more information on the abnormal regions. Watershed method to segment these regions is one of the widely used techniques. The watershed algorithm is sensitive to minor changes in the regions. This sensitivity and the presence of noisy pixels make the algorithm to suffer from over-segmentation. This paper presents an effective pre-segmentation approach to minimise over-segmentation by segmenting the affected region from the normal tissues.
\end{abstract}

Keywords: Stroke, MRI, Segmentation, Watershed Algorithm, DWI, ADC

\section{INTRODUCTION}

According to WHO reports, stroke ranks second in morbidity next to ischaemic heart disease[1]. Stroke is a cerebrovascular event that affects the supply of blood to the brain cells. Strokes are classified as Ischemic Stroke, caused due to block of blood, and Haemorrhagic stroke, caused by breaks and bleeds in blood vessel [2]. It causes paralysis on specific organ of the body, can also lead to memory loss, vision problems, speech problem. In order to diagnose stroke regions the diffusion weighted imaging sequence employed in MR imaging is considered to be the gold-standard method to identify acute stroke and is popularly known as stroke sequence as it provides sensitivity of over $90 \%$ and specificity over $95 \%$ of the stroke cases [3]. Since the stroke is related to diffusion of water molecules in the brain cells a diffusion coefficient mapping will yield more accurate results. Thus DWI images along with ADC mapping is employed clinically to diagnose strokes. The location of stroke in brain has significant clue on the effect it does on the patients. The segmentation of the affected region is to be done accurately for proper treatment plan.

Segmentation of an image is a mid-level processing technique which divides the image into meaningful parts based on certain test conditions. There are various methods available for segmentation, based on either discontinuity or on similarity measures of the pixel characteristic. Watershed algorithm is the hybrid of discontinuity and similarity based segmentation. It was first proposed by Meyer[4] in 1970 and is still used as a powerful tool for segmenting images. The major limitation inherent in watershed algorithm is oversegmentation. The use of markers as priori to the segmentation yields better results to overcome oversegmentation. In medical images the shape of the abnormality is also crucial and should not be altered by the application of image processing techniques. Morphological operations are the powerful tools employed in image processing for retaining the shapes of objects [5]. Marker controlled watershed along with morphological operations ensures proper segmentation and preserves the shape of the segmented regions.

The paper is organised as follows; section II gives introduction to necessary theory on the important tools used is given, in section III the methodology employed to reduce the over-segmentation in watershed algorithm is discussed, section IV presents results and section $\mathrm{V}$ highlights the discussion on the obtained results.

\section{MATERIALS AND METHODS:}

\subsection{MRI and DWI Sequence:}

Magnetic Resonance Imaging (MRI) is one of the chief imaging modality used to obtain the images of internal body parts. Among the medical imaging techniques, this mode of image acquisition uses least harmful radiations to acquire images and hence said to be non-hazardous. A conventional diagnosis uses $\mathrm{T} 1$ and $\mathrm{T} 2$ sequences to find abnormalities. But these sequences are not helpful for diagnosing strokes on the onset[3]. 


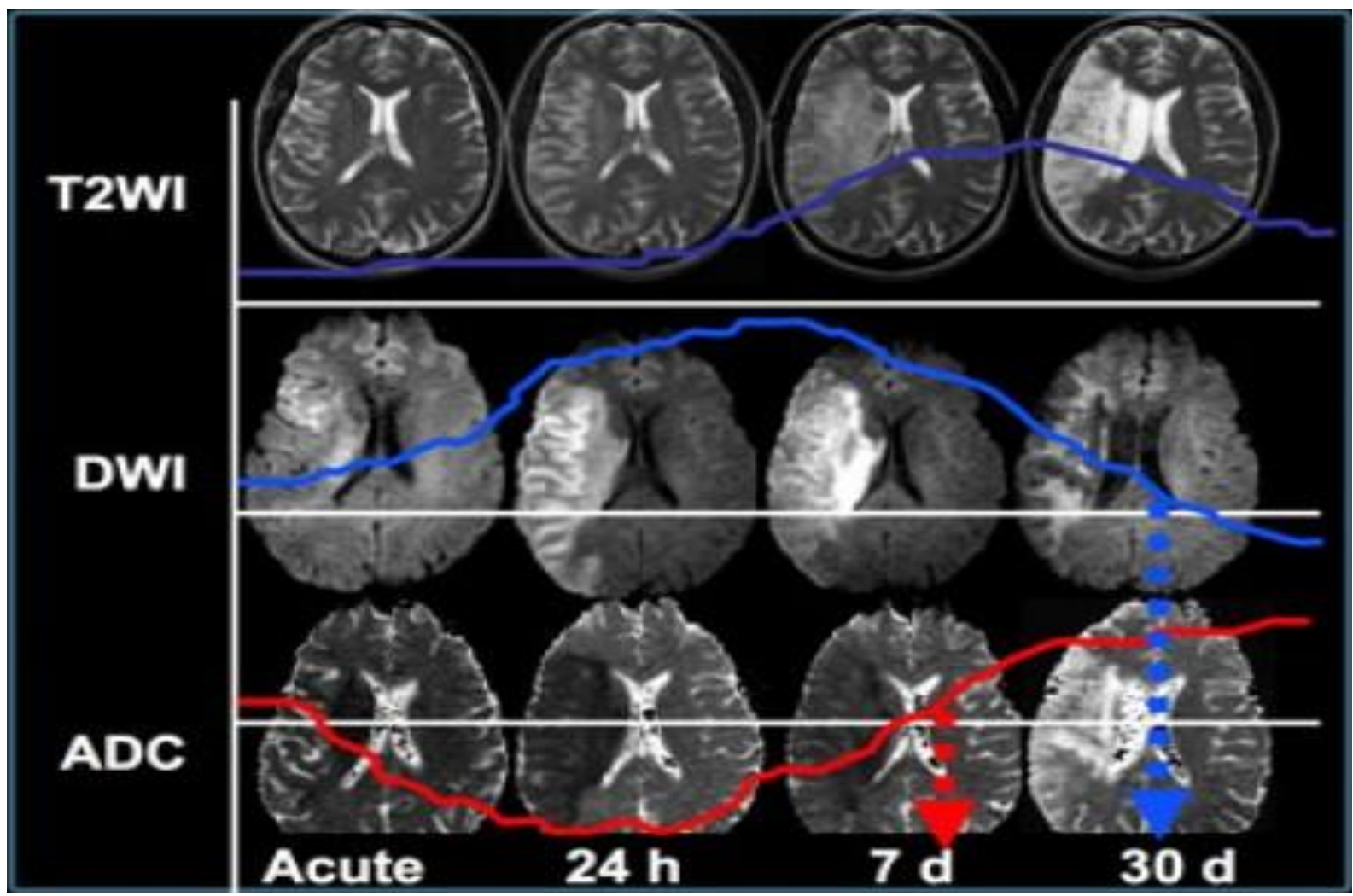

Fig. 1 Comparison of T2 weighted imaging sequence with DWI and ADC Image courtesy: www.radiologyassistant.nl

As a solution to this, the image construction algorithms are employed that uses the Brownian motion of fluids in the body to acquire images. The sequence thus obtained using Brownian energy is called diffusion weighted imaging (DWI) sequence. This sequence produces faithful images as early as few minutes from the onset of stroke and commonly called as stroke sequence. The variation in the diffusion rate of water molecules is the key feature to highlight anomalies in the acquired images in the DWI sequence. During stroke, the essential fluids along with water are blocked to the cells. This alters the Brownian energy associated with water molecules. The area of restricted diffusion that corresponds to stroke activity, appears bright in DWI sequence [6].

The DWI sequence alone is not preferred to confirm the stroke by experts. Additionally, a diffusion mapping, called apparent diffusion coefficient (ADC) mapping is done, where the result of mapping can be expressed as signal intensity or as an image[7]. The ADC mapping is a confirmative test to diagnose the cerebrovascular accident. The region observed as hyper-intense in DWI should appear hypo-intense in ADC mapping i.e. a HIGH on DWI should appear LOW in ADC. The following figure shows a stroke region as observed in DWI and ADC images.

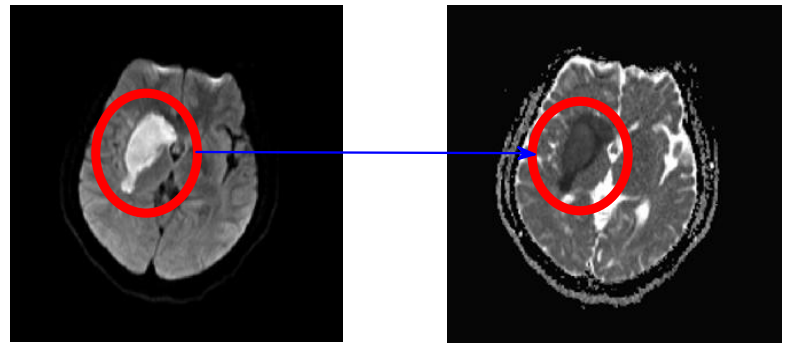

Fig. 2 DWI and ADC Sequences highlighting abnormality

\subsection{Morphological Operations}

Morphological operations are the set based functions that use logical relations for their operation. These are basically used to remove the imperfections in the images while retaining the shapes of the object. Morphological operations use structuring elements to perform the specified action. The structuring element is basically a sub-image of very small size that usually consists of binary values. The basic operations that are used are erosion and dilation. Any other morphological operation is a combination of dilation and erosion operations. The erosion of an image A by structuring element $B$ is the set of all points $\mathrm{Z}$ such that the structuring element $\mathrm{B}$ is translated by $\mathrm{z}$, is a subset of the image and is defined as

$$
A \ominus B=\left\{z \mid(B)_{z} \subseteq A\right\}
$$

The dilation of an image A (set) by structuring element $B$ is defined as

$$
A \oplus B=\left\{z \mid \widehat{(B)}_{z} \cap A \neq \emptyset\right\}
$$


If set $B$ is reflected about its origin and shifted by $\mathrm{z}$, then the dilation of $\mathrm{A}$ by $\mathrm{B}$ is the set of all displacements $\mathrm{z}$ such that $\mathrm{B}^{\wedge}$ and $\mathrm{A}$ have at least one common element[8].

In our work, morphological operations are used in preprocessing operation like skull stripping. Skull stripping is a process of removing non-brain regions like skull, scalp and meninges. Roslan et al. have shown that the skull stripping using double threshold morphological operations yields better result than Otsu's thresholding method [9]. The structuring element used in our work is a 'disk' of suitable size. A combination of few other morphological operations is used to achieve better performance.
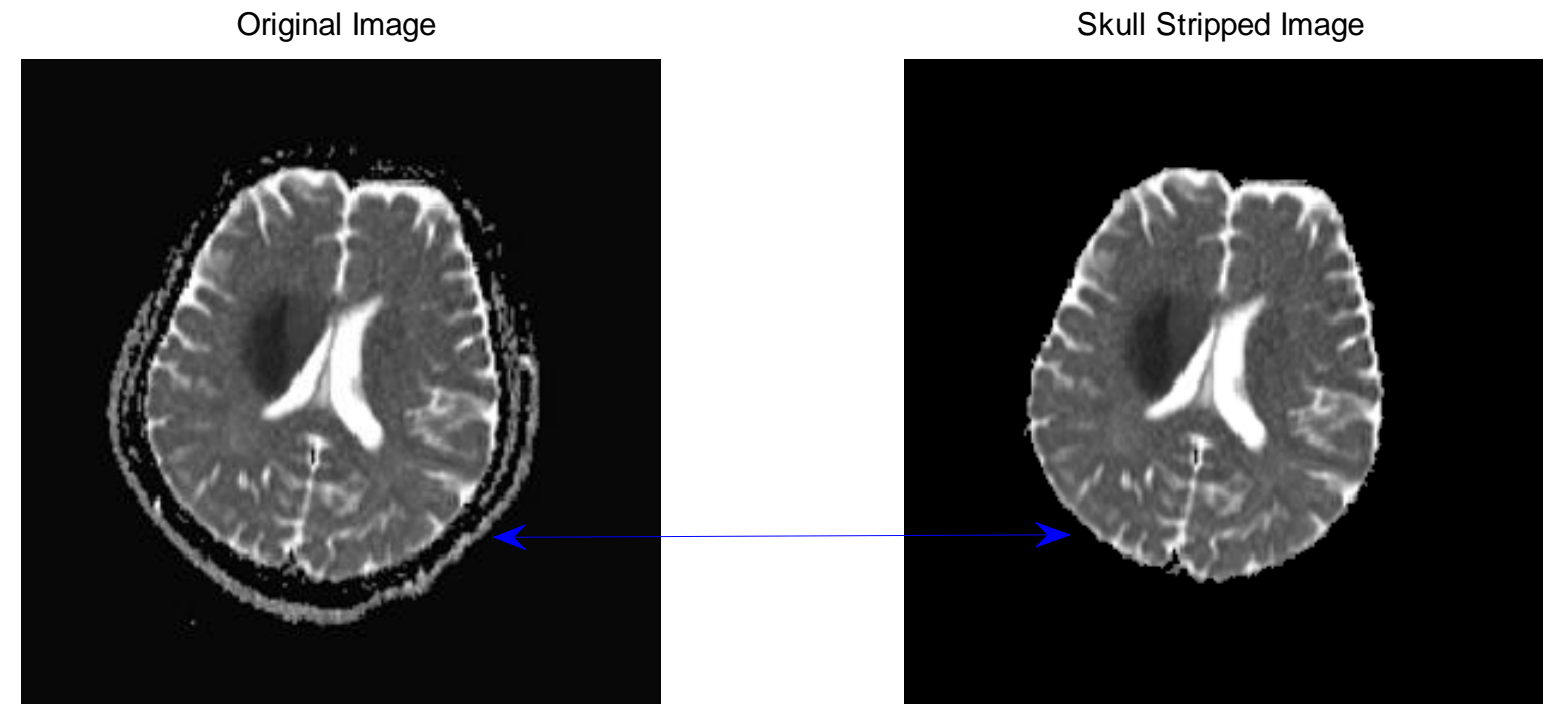

Fig.3 Skull stripping using morphological

\subsection{Watershed Algorithm}

Watershed algorithm is basically a segmentation technique that depends on region of similarity[10] and is a powerful tool for image segmentation. In [5]it is also considered as the hybrid approach of region and edge based techniques which uses thresholding operations indirectly. The watershed algorithm depends mainly on the image intensities, using local maxima/minima the algorithm tries to segment the image into several connected components [11]. The advantage of watershed algorithm is that it is automatic in nature and is computationally efficient. The major drawback of it is over-segmentation. The over-segmentation problem is effectively addressed by the using morphological markers prior to application of watershed algorithm. The markers are set of pixels that belong to object and background which are used to give a priori knowledge on 'regions' to be segmented.

By defining proper number of markers the segmentation can be controlled. The following figure illustrates the effectiveness of markers to limit the number of segmented regions using watershed algorithm
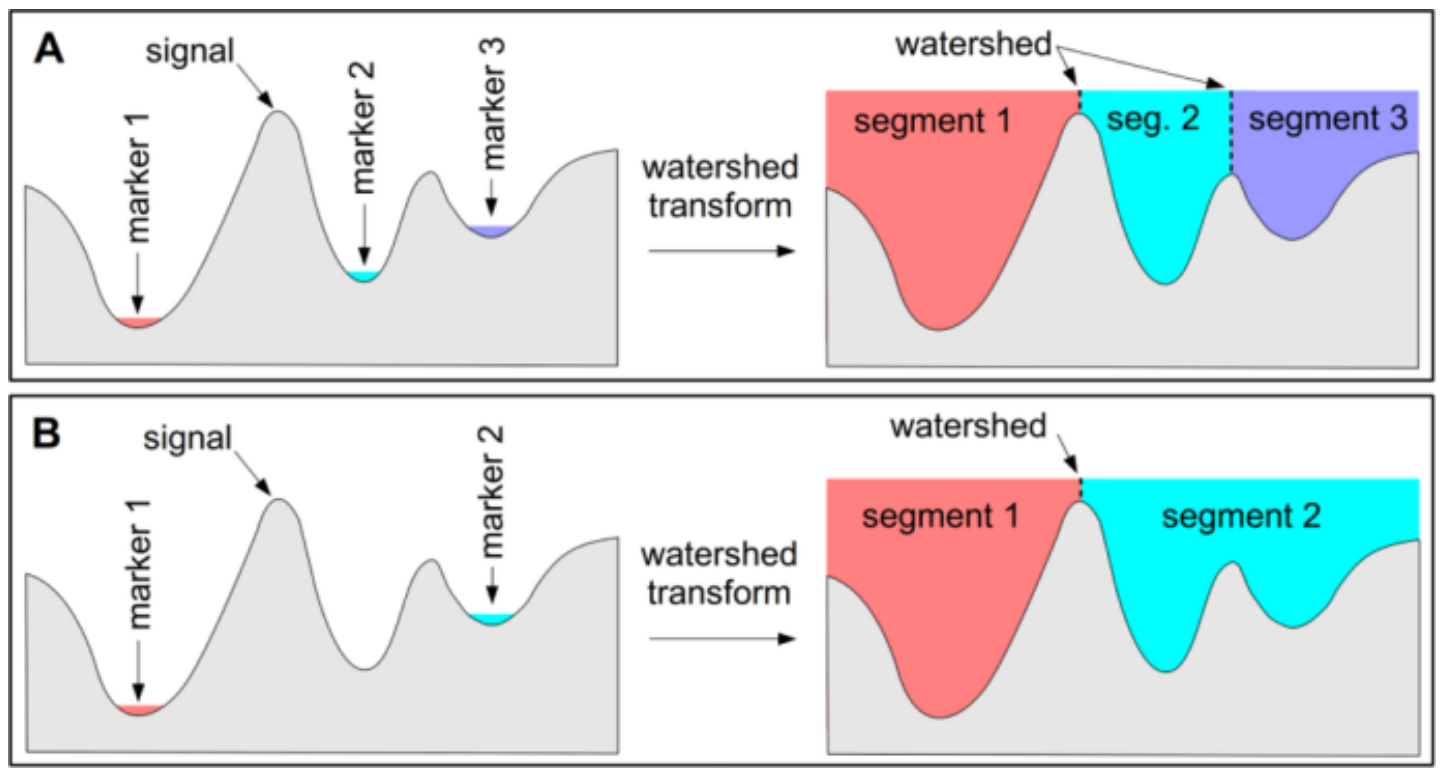

Fig. 4 Illustration of marker controlled segmentation 
The watershed algorithm is usually implemented either by flood based method or by topographical distance method [12][13]. In this paper, Meyer's flood based method is employed.

The watershed method of segmenting an image uses catchment basins and watershed lines to mark a region. The catchment basin is the set of pixels which are connected to local minima of the region. The watershed lines are those which separate two catchment basins.

In flood based method, minima (lowest intensity pixel) of a catchment basin (region) acts as starting point from which the flooding starts towards the highest topographical surface (local maxima). When the flood reaches top of the surface (maxima), it tends to merge with the neighbouring catchment basin (region). At this point, a watershed line (boundary line) is drawn between the two catchment basins to avoid merging and this action results in construction of dam. The flooding process happens simultaneously over entire image and only dams are visible at the end of segmentation. Generally, the flooding operation is done on the gradient of the image instead of image itself.

\section{PROPOSED METHOD:}

The literatures suggest that the over-segmentation inherent in watershed algorithm is due to presence of noisy pixels. So by using noise filters at the pre-processing stages the oversegmentation can be reduced significantly. The proposed method uses pre-processing operations to enhance the quality of the image by filtering noises if present any. Since the proposed method uses watershed algorithm, the gradient of the filtered image should be calculated. DWI sequence in MRI is a clear intensity image sequence where the normal and abnormal regions can be distinguished through their intensity profile. Thus the filtered image is thresholded with suitable value to get members for abnormal regions. It is observed that the abnormal regions possess higher intensity levels in DWI sequence. Thus threshold value is chosen at higher point on the gray scale.

The threshold image is then correlated with original image to extract possible abnormal regions. The procedure is repeated for ADC images and the threshold values are chosen at the lower range since the abnormality is hypo-intense in ADC mapping. The extracted regions are spatially correlated to obtain a single region that highlights abnormality. From the obtained image the spatial locations are marked and corresponding pixels from DWI image is isolated. The region thus obtained is subjected to morphological operations to retain their shapes before applying watershed algorithm. The watershed algorithm forms region within and are labelled using HSV colour mode. The result of watershed algorithm is post processed to make it visually appealing; this gives more details on the abnormal region in an image.

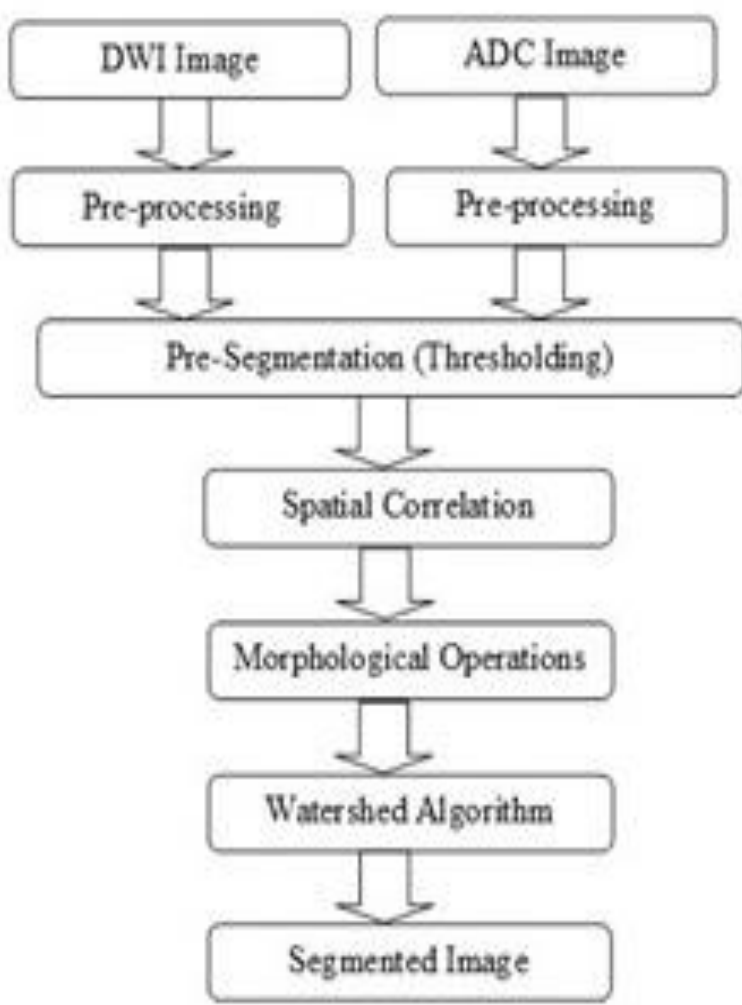

\section{Fig.5 Flow chart of proposed algorithm}

\section{RESULTS:}

The proposed algorithm is implemented on several test images using MatLab2013a software on Intel(R) Pentium(R)4 CPU 2.80GHz $1 \mathrm{~Gb}$ 32-bit OS system. The test images were selected from the database created on own obtained from the Department of Radiology, K.S.Hegde Hospital, Mangalore. For better visual clearance the segmented images are labelled in colour. The segmented images have been overlapped on the original image for ease of comparison. Since the proposed algorithm extracts specific regions from the original image and then the regions are subjected to watershed segmentation the major drawback of over-segmentation is reduced considerably. For the segmented images statistical parameters like PSNR and MSE are calculated along with the area of the obtained region. The statistical parameters calculated for the segmented image are given below and the area of the segmented region in terms of pixels is also tabulated.

Table 1: Statistical Parameters of Segmented Image

\begin{tabular}{|c|c|c|c|}
\hline Images & MSE & PSNR & Area \\
\hline 1 & 0.3077 & 53.2833 & 4226 \\
\hline 2 & 0.1397 & 56.7113 & 9085 \\
\hline 3 & 0.2554 & 54.0932 & 5481 \\
\hline 4 & 0.1122 & 57.6660 & 11094 \\
\hline 5 & 0.2500 & 54.1855 & 4802 \\
\hline 6 & 0.5584 & 50.6956 & 2277 \\
\hline
\end{tabular}




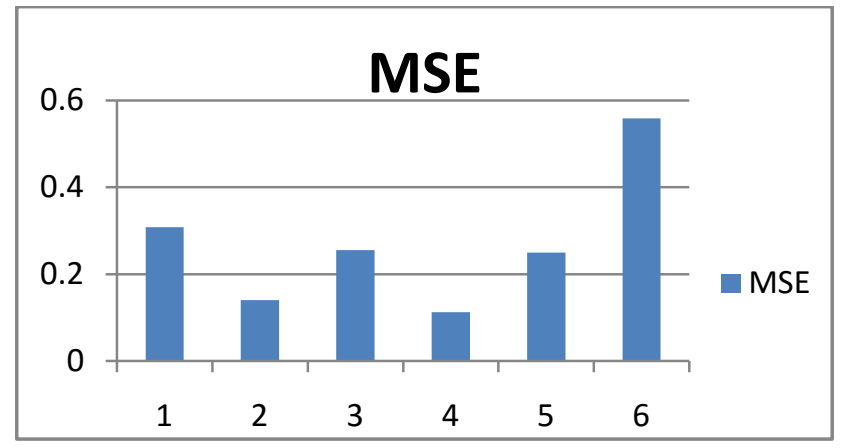

Chart-1: Mean Square Error

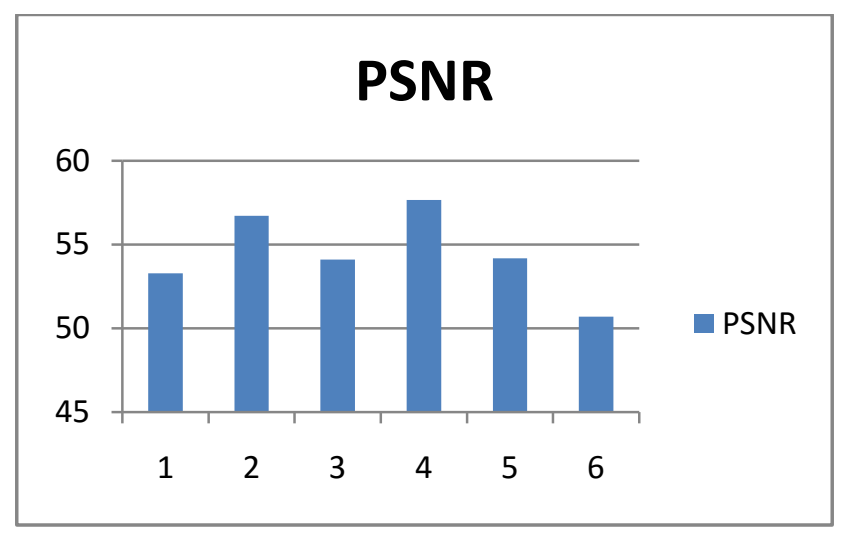

Chart-1: Peak Signal to Noise Ratio
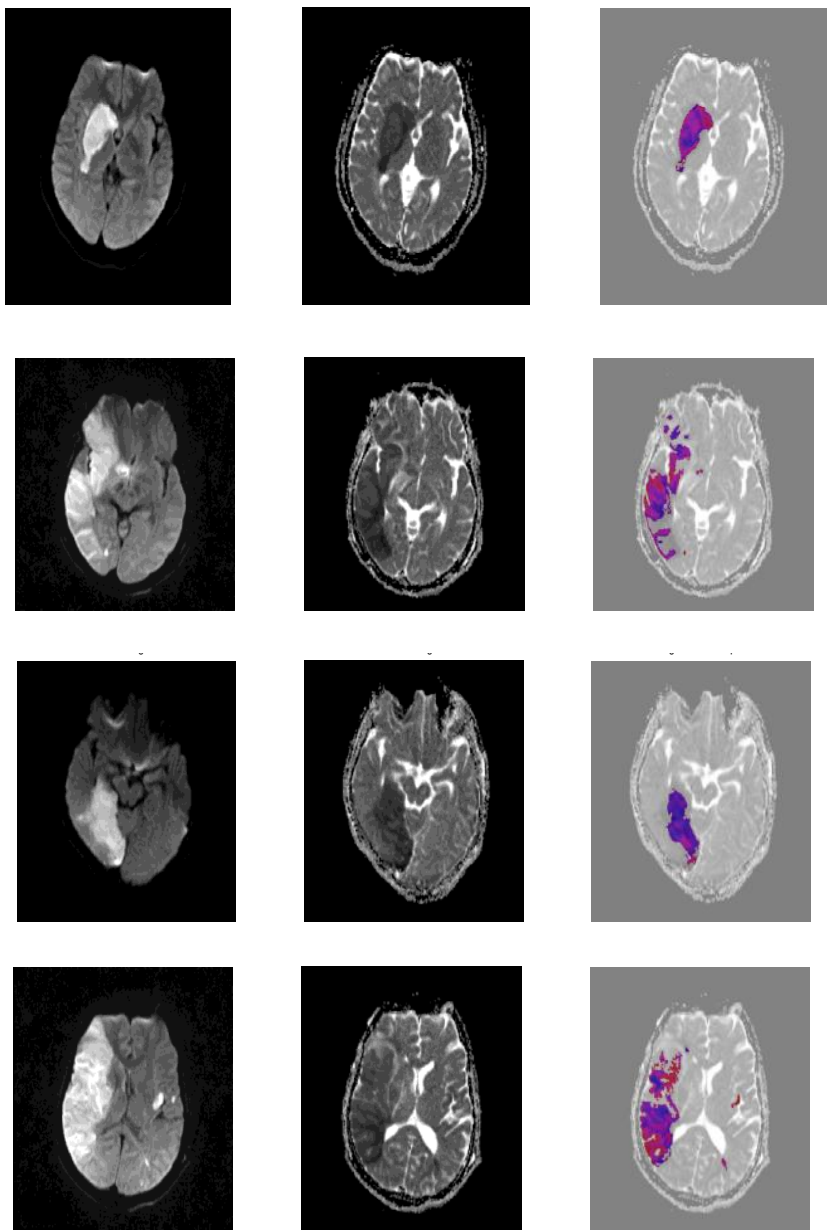
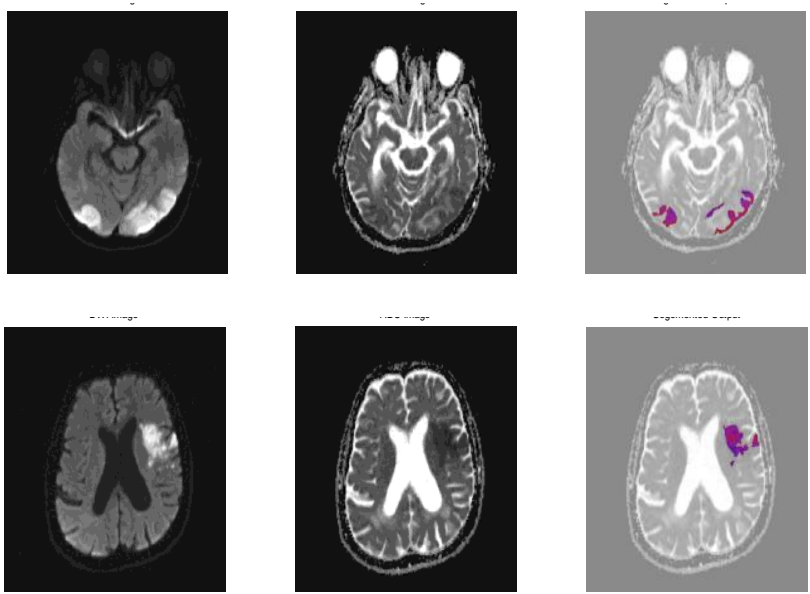

Fig. 6: Result of the proposed method

First column corresponds to DWI sequence; second column corresponds to ADC sequence; third column corresponds to the results obtained by using proposed method.

\section{DISCUSSION}

The DWI and ADC images were separately considered for pre-processing operations. Since the images are chosen manually care should be taken while choosing an image slice. The slice number of ADC should be same as DWI. The noise is one of the prime factors that lead to over-segmentation while using watershed algorithm. If noise filtering is employed at the pre-processing stage, over-segmentation can be effectively controlled. In this paper a new technique is tried successfully to limit the segmentation to region of interest. To achieve this, firstly non-brain regions were removed from the original image using skull stripping technique by employing morphological operations. A subimage called structuring element is employed for the purpose. It is observed that the shape and size of structuring elements plays vital role for skull stripping. Pre-segmentation process includes threshold operation which helps to extract possible members for region of interest (ROI). This significantly reduces over-segmentation since the regions in pre-segmented image is very less compared to original image. This semisegmented image is subjected to flood-based watershed algorithm to obtain the segmented image. The resultant images showed good statistical performance in terms of mean-square error and peak-signal-to-noise ratio. Colour labelling is done to identify the dead cells and penumbra. Colour coding is done such that dead cells are coloured with red and penumbra with blue.

\section{CONCLUSION}

In our work DWI and ADC images are pre-processed separately and the images obtained after pre-segmentation are spatially correlated to obtain a single image that has only ROI. Since the stroke in brain is not uniformly distributed, we have used colour labelling technique to differentiate low and high intensities within the region. The red colour in the labelled image represents higher intensity and blue/purple represents lower intensities. 
We conclude that the application of pre-processing techniques like noise filtering and skull stripping along with the threshold based pre-segmentation considerably reduces over-segmentation of watershed algorithm. For skull stripping, the disk structure gives good results than other shapes.

\section{REFERENCES}

[1] "WHO | The top 10 causes of death."

[2] "Stroke." National Library of Medicine.

[3] "Diffusion weighted MRI in acute stroke | Radiology Reference Article | Radiopaedia.org." [Online]. Available: http://radiopaedia.org/articles/diffusion-weighted-mri-inacute-stroke-1. [Accessed: 03-Jul-2015].

[4] S. Beucher and C. Lantuejoul, "Use of Watersheds in Contour Detection," International Workshop on Image Processing: Real-time Edge and Motion Detection/Estimation. pp. 12-21, 1979.

[5] R. Gonzalez and R. Woods, Digital image processing. 2002.

[6] "MRI for Neurology." [Online]. Available: http://neuro.wustl.edu/files/6413/4461/5738/Week_3_Hando ut_MRI_for_Neurology.pdf. [Accessed: 04-Aug-2015].

[7] "Apparent diffusion coefficient | Radiology Reference Article | Radiopaedia.org." [Online]. Available: http://radiopaedia.org/articles/apparent-diffusion-coefficient1. [Accessed: 10-Aug-2015].

[8] S. Ravi and A. M. Khan, "Morphological Operations for Image Processing : Understanding and its Applications."

[9] R. Roslan, N. Jamil, and R. Mahmud, "Skull Stripping of MRI Brain Images using Mathematical Morphology," IEEE EMBS Conf. Biomed. Eng. Sci., no. December, pp. 26-31, 2010.

[10] J. Roerdink and a Meijster, "The Watershed Transform: Definitions, Algorithms and Parallelization Strategies," Fundam. Informaticae, vol. 41, no. 1-2, pp. 187228, 2000.

[11] F. Ségonne, a. M. Dale, E. Busa, M. Glessner, D. Salat, H. K. Hahn, and B. Fischl, "A hybrid approach to the skull stripping problem in MRI," Neuroimage, vol. 22, no. 3, pp. 1060-1075, 2004.

[12] F. Meyer, "Topographic distance and watershed lines," Signal Processing, vol. 38, no. 1, pp. 113-125, 1994.

[13] S. Larnier, J. Fehrenbach, and M. Masmoudi, "The Topological Gradient Method: From Optimal Design to Image Processing," Milan J. Math., 2012. 